\title{
Effects of brain-derived neurotrophic factor and neurotrophin-3 on the neuronal differentiation of rat adipose-derived stem cells
}

\author{
WENCHEN JI ${ }^{1}$, XIAOWEI ZHANG ${ }^{1}$, LE JI $^{2}$, KUNZHENG WANG ${ }^{3}$ and YUSHENG QIU ${ }^{1}$ \\ ${ }^{1}$ Department of Orthopedics, The First Affiliated Hospital of Xi'an Jiaotong University, Xi'an, Shaanxi 710061; \\ ${ }^{2}$ Department of Orthopedics, The Third Affiliated Hospital of Xi'an Jiaotong University, Xi'an, Shaanxi 710068; \\ ${ }^{3}$ Department of Orthopedics, The Second Affiliated Hospital of Xi'an Jiaotong University, Xi'an, Shaanxi 710004, P.R. China
}

Received October 1, 2014; Accepted June 23, 2015

DOI: $10.3892 / \mathrm{mmr} .2015 .4099$

\begin{abstract}
Tissue engineering is a promising method that may be used to treat spinal cord injury (SCI). The underlying repair mechanism of tissue engineering involves the stable secretion of neurotrophins from seed cells, which eventually differentiate into neurons; therefore, the selection of appropriate seed cells, which stably secrete neurotrophins that easily differentiate into neurons requires investigation. Adipose-derived stem cells (ADSCs), which are adult SCs, are advantageous due to convenience sampling and easy expansion; therefore, ADSCs are currently the most popular type of seed cell. Brain-derived neurotrophic factor (BDNF) and neurotrophin-3 (NT-3) possess superior properties, when compared with other neurotrophic factors, in the maintenance of neuronal survival and promotion of SC differentiation into neurons. The present study used two lentiviruses, which specifically express BDNF and NT-3 [Lenti-BDNF-green fluorescent protein (GFP), Lenti-NT-3-red fluorescent protein (RFP)], to transfect third-generation ADSCs. Three types of seed cell were obtained: i) Seed cells overexpressing BDNF (ADSC/Lenti-BDNF-GFP); ii) seed cells overexpressing NT-3 (ADSC/Lenti-NT-3-RFP); and iii) seed cells overexpressing BDNF and NT-3 (ADSC/Lenti-BDNF-GFP and NT-3-RFP). The transfected cells were then induced to differentiate into neurons and were divided into a further four groups: i) The BDNF and NT-3 co-overexpression group; ii) the BDNF overexpression group; iii) the NT-3 overexpression group; and iv) the control group, which consisted of untransfected ADSCs. The results of the present study demonstrate that BDNF and NT-3 expression was higher 10 days after induction, as detected by reverse transcription-quantitative
\end{abstract}

Correspondence to: Professor Yusheng Qiu, Department of Orthopedics, The First Affiliated Hospital of Xi'an Jiaotong University, 277 Yanta West Road, Xi'an, Shaanxi 710061, P.R. China E-mail: yushengqiu1957@163.com

Key words: brain-derived neurotrophic factor, neurotrophin-3, adipose-derived stem cells, neuronal differentiation polymerase chain reaction (RT-qPCR) and western blotting. Neuron-specific enolase is a neuronal marker, the expression of which was highest in the BDNF and NT-3 co-overexpression group, followed by the BDNF overexpression group and then by the NT-3 overexpression group. The lowest expression levels of NSE were detected in the control group, as determined by RT-qPCR, western blotting and immunofluorescent staining. These results indicate that BDNF and NT-3 exert a synergistic effect, which may promote the neuronal differentiation of ADSCs. The present study provides a solid theoretical foundation for future experiments regarding the use of tissue engineering technology for the treatment of SCI.

\section{Introduction}

Spinal cord injury (SCI) usually leads to permanent loss of function, resulting in marked economic burden to the family of the individual, as well as society as a whole. There is currently no effective therapeutic method for the treatment of SCI $(1,2)$. Since the development of tissue engineering technology in the biomedical field, numerous studies have investigated the possibility of using tissue engineering to repair SCI $(3,4)$. The underlying repair mechanism of tissue engineering is the secretion of neurotrophins from seed cells, and the eventual differentiation of the cells into neurons. Therefore, the selection of appropriate seed cells, which stably secrete neurotrophins and easily differentiate into neurons, is an important topic of discussion $(5,6)$. Seed cells include non stem cells (SCs), such as fibroblasts; and SCs, which may be either non-adult SCs, such as neural and embryonic SCs, or adult SCs, such as adipose-derived SCs (ADSCs) and muscle-derived SCs. Compared with non-SCs, $\mathrm{SCs}$ are regarded as superior; whereas compared with non-adult SCs, adult SCs are regarded as superior (7), particularly ADSCs, which are advantageous due to convenience sampling and easy expansion. ADSCs are currently the most popular type of seed cells, and previous studies have demonstrated their ability to secrete neurotrophins and differentiate into neurons; however, these abilities are limited $(8,9)$. Gene therapy has been proposed as a method to overcome this problem via transfection of the necessary genes into the cells resulting in their consistent expression, which may prompt the differentiation of ADSCs into neurons (10). Brain-derived 
Table I. Primer sequences.

Neurotrophin-3-ClaI

Neurotrophin-3-SpeI

Brain-derived neurotrophic factor-ClaI

Brain-derived neurotrophic factor-SpeI
Forward,AATCGATATGTCCATCTTGTTTTATGTGATA Reverse, AACTAGTTCATGTTCTTCCGATTTTTCTCGA Forward, AATCGATATGACCATCCTTTTCCTTACTATG Reverse, AACTAGTTTATCTTCCCCTTTTAATGGTCAA neurotrophic factor (BDNF) and neurotrophin (NT)-3 possess superior properties, as compared with other types of neurotrophic factors, in the maintenance of neuronal survival and the promotion of SC differentiation into neurons $(11,12)$; however, the synergistic effects of BDNF and NT-3 on the neuronal differentiation of ADSCs have yet to be elucidated. The present study constructed lentiviruses specifically expressing BDNF and NT-3, and used these to co-transfect ADSCs. The effects of BDNF and NT-3 on the neuronal differentiation of ADSCs were then determined. The present study aimed to provide a foundation for future experiments regarding the suitable selection of seed cells for use in tissue engineering for the treatment of SCI.

\section{Materials and methods}

Experimental animals. Two female Sprague-Dawley rats, (age, 1 month; weight, $150 \pm 30 \mathrm{~g}$ ) were provided by the Centre of Experimental Animals, School of Medicine, Xi'an Jiaotong University (Xi'an, China). The rats were maintained in a controlled environment $\left(18-26^{\circ} \mathrm{C} ; 40-70 \%\right.$ relative humidity), and were given ad libitum access to food and water. All the experiments of the present study were approved and supervised by the Ethics Committee of the Xi'an Jiaotong University.

Isolation, culture and identification of ADSCs. Chloral hydrate $(0.1 \mathrm{ml} / 100 \mathrm{~g}$; The Second Affiliated Hospital of Xi'an Jiaotong University) was used to anesthetize the rats. Their adipose tissue $(1 \times 1 \mathrm{~cm})$ was then harvested, sectioned and incubated for $0.5 \mathrm{~h}$ with $0.1 \%$ collagenase type I (Sigma-Aldrich, St. Louis, MO, USA) in a centrifuge tube containing Dulbecco's modified Eagle's medium (DMEM)/F12 supplemented with $10 \%$ fetal bovine serum (FBS; Hyclone, GE Healthcare, Logan, UT, USA). The tissue was subsequently centrifuged at $2,000 \times \mathrm{g}$ for $10 \mathrm{~min}$, the supernatant was discarded and phosphate-buffered saline (PBS) was added, prior to further centrifugation at $1,000 \mathrm{xg}$ for $10 \mathrm{~min}$. The sediment was then suspended in DMEM/F12 supplemented with $10 \%$ FBS, the cell density was adjusted to $1 \times 10^{5} / \mathrm{ml}$, and the cells were seeded in a $25-\mathrm{cm}^{2}$ cell culture flask. Once the original generation of cells reached 90\% confluence, the entire medium was aspirated, and the cells were washed with PBS to remove any tissue fragments and blood cells. Subsequently, 0.25\% trypsin/0.02\% EDTA solution was added to the cells for 3 min for digestion, and DMEM/F12 was added to terminate the digestion. The cells were then centrifuged at $1,000 \mathrm{x} g$ for $6 \mathrm{~min}$. The supernatant was aspirated and the cells were suspended in DMEM/F12 supplemented with $10 \%$ FBS, the cells were passaged at a ratio of $1: 3$, adjusted to a density of $1 \times 10^{5} / \mathrm{ml}$ and cultured until they had reached $90 \%$ confluence, which usually takes $\sim 72 \mathrm{~h}$ for first-generation cells.

Third-generation cells were used in the present study, and were seeded at a density of $1 \times 10^{5} /$ well in 6 -well plates. The cells were then divided into the experimental and control groups. Once the cells had reached $80 \%$ confluence, the experimental group was placed into osteogenic induction media, consisting of $0.1 \mu \mathrm{mol} / 1$ dexamethasone (Sigma-Aldrich), $10 \mathrm{mmol} / 1 \beta$-glycerophosphate sodium (Sigma-Aldrich), $50 \mathrm{mg} / \mathrm{l}$ vitamin C (Sigma-Aldrich), $0.01 \mu \mathrm{mol} / 1$ vitamin $\mathrm{D}_{3}$ (Sigma-Aldrich) and $10 \% \mathrm{FBS} / \mathrm{low}$ glucose-DMEM. The control group continued to be cultured in DMEM/F12 supplemented with 10\% FBS. After 2 and 4 weeks, alkaline phosphate (ALP) and alizarin red staining were performed, respectively (13).

Construction of lentivrius and transfection of ADSCs. Total RNA was extracted from the U87 human glioblastoma cell line (Sigma-Aldrich) using RNAiso (Takara Bio, Inc., Otsu, Japan), and cDNA was synthesized by reverse transcription (RT) using an RT kit (Promega Corporation, Madison, WI, USA). Polymerase chain reaction (PCR) was conducted using a PCR Amplifier (cat. no. 070-851; Biometra GmbH, Göttingen, Germany) to amplify the gene expression of BDNF and NT-3. The primers used are listed in Table I (Sangon Biotech Co., Ltd., Shanghai, China). The PCR cycling conditions were as follows: $94^{\circ} \mathrm{C}$ for $30 \mathrm{sec}$, followed by 30 cycles at $98^{\circ} \mathrm{C}$ for $10 \mathrm{sec}, 55^{\circ} \mathrm{C}$ for $15 \mathrm{sec}$ and $72^{\circ} \mathrm{C}$ for $45^{\circ} \mathrm{C}$, and a final extension step at $72^{\circ} \mathrm{C}$ for $45 \mathrm{sec}$. Following PCR, BDNF and NT-3 were subcloned into pLV-CMV-EF1a-green fluorescent protein (GFP) and pLV-CMV-EF1a-red fluorescent protein (RFP) expression lentivectors, respectively. Subsequently, 293 T cells (cat. no. ab95494; Abcam, Cambridge, MA, USA) were transfected with $\mathrm{pLV} /$ helper packaging plasmid mix, containing either pLV-CMV-EF1a-BDNF-GFP or pLV-CMV-EF1a-NT-3-RFP expression lentivector (40 $\mu \mathrm{g})$, and pMD2.G (20 $\mu \mathrm{g}$; Invitrogen Life Technologies, Carlsbad, CA, USA) and psPAX2 (20 $\mu \mathrm{g}$; Invitrogen Life Technologies) at a ratio of $2: 1: 1$. Finally, two types of lentivirus, Lenti-BDNF-GFP and Lenti-NT-3-GFP were obtained.

The third-generation ADSCs were inoculated at a density of $75-80 \%$, and then transfected with the lentiviruses at a multiplicity of infection (MOI) of 1, 10 and 100, in order to determine the best MOI value in each group. Three cell groups were generated: i) The Lenti-BDNF-GFP transfected group; ii) the Lenti-NT-3-RFP transfected group; and iii) the Lenti-BDNF-GFP and Lenti-NT-3-RFP co-transfected 
group. The efficiency of lentiviral gene transfer into ADSCs was determined according to the presence of fluorescent cells, as detected by fluorescence microscopy (DMI4000B; Leica Microsystems GmbH, Wetzlar, Germany) 72 h post-transfection. After determining the suitable MOI, flow cytometry (cat. no. 175487; Beckman Coulter, Inc., Brea, CA, USA) was used to detect the expression of GFP in the Lenti-BDNF-GFP transfected group, the expression of RFP in the Lenti-NT-3-RFP transfected group, and the co-expression of GFP and RFP in the Lenti-BDNF-GFP and Lenti-NT-3-RFP co-transfected group.

Induction of the transfected cells into neural cells. Neural induction media containing 10\% FBS/L-DMEM, $10 \mu \mathrm{mol} / 1$ forskolin, $5 \mathrm{mmol} / 1 \mathrm{KCl}, 2 \mathrm{mmol} / 1$ sodium valproate, $1 \mathrm{~mol} / 1$ hydrocortisone and $5 \mathrm{mg} / \mathrm{l}$ insulin (all Sigma-Aldrich) was used to induce the differentiation of transfected ADSCs into neurons. The ADSCs were divided into four groups: i) The BDNF and NT-3 co-overexpression group (BDNF and NT-3 co-transfected ADSCs); ii) the BDNF overexpression group (BDNF transfected ADSCs); iii) the NT-3 overexpression group (NT-3 transfected ADSCs); and iv) the control group (untransfected ADSCs). After 10 days, the identification of BDNF, NT-3 and neuron-specific enolase (NSE) was conducted using immunofluorescent staining, RT-quantitative (q)PCR and western blotting.

Immunofluorescent staining. Medium was removed from the cells and they were fixed with $4 \%$ paraformaldehyde for 30 min. A total of $30 \% \mathrm{H}_{2} \mathrm{O}_{2}$ was used to block endogenous peroxidase activity, and the cells were subsequently incubated with $0.3 \%$ Triton-X 100 (Sigma-Aldrich) for $20 \mathrm{~min}$ at room temperature. The cells were then incubated with rabbit polyclonal anti-NSE (1:50 dilution; cat. no. ab53025; Abcam) at $4^{\circ} \mathrm{C}$ overnight. The cells were incubated with a fluorescein isothiocyanate-conjugated goat anti-rabbit immunoglobulin $\mathrm{G}$ secondary antibody (1:100 dilution; cat. no. ab6717; Abcam) for $4 \mathrm{~h}$ at room temperature. Finally, the cells were incubated with $100 \mu \mathrm{g} / 1$ DAPI for $15 \mathrm{~min}$. Fluorescence microscopy was used (magnification, x200) to observe the positively-stained cells in each group. Three wells were selected, and five fields from each were used to calculate the percentage of positive cells (stained red) from the number of total cells (blue-stained nuclei). The experiment was repeated three times.

RT-qPCR to detect BDNF, NT-3 and NSE mRNA expression levels. Total RNA was extracted from the transfected ADSCs using TRIzol ${ }^{\circledR}$ reagent (Invitrogen Life Technologies), and was reverse transcribed using oligo (dT) primers, according to the manufacturer's instructions (Takara Bio, Inc.). RT-qPCR was conducted using a PCR Amplifier (cat. no. 070-851; Biometra $\mathrm{GmbH}$ ) and SYBR Green (cat. no. 4367659; Invitrogen Life Technologies). The PCR cycling conditions were as follows: $94^{\circ} \mathrm{C}$ for $30 \mathrm{sec}$, followed by 40 cycles at $98^{\circ} \mathrm{C}$ for $10 \mathrm{sec}$ and $60^{\circ} \mathrm{C}$ for $20 \mathrm{sec}$, and a final step at $72^{\circ} \mathrm{C}$ for $20 \mathrm{sec}$. The primer pairs for BDNF, NT-3, NSE and GAPDH (which served as a housekeeping gene) are listed in Table II (Sangon Biotech Co., Ltd.). The expression levels of the genes of interest were determined according to the standard curve, using the $2^{-\Delta \Delta \mathrm{Ct}}$ quantification method (14).
Table II. Primer sequences.

\begin{tabular}{ll}
\hline Primer & \multicolumn{1}{c}{ Primer sequence (5'-3') } \\
\hline NT-3 & Forward, CGTGGTGGCGAACAGAACAT \\
NT-3 & Reverse, GGCCGATGACTTGTCGGTC \\
BDNF & Forward, CTACGAGACCAAGTGCAATCC \\
BDNF & Reverse, AATCGCCAGCCAATTCTCTTT \\
NSE & Forward, CGAGGACACGTTCATTGCAGA \\
NSE & Reverse,GAGCTGGTTGTACTTCGCCAGAC \\
GAPDH & Forward, GCACCGTCAAGGCTGAGAAC \\
GAPDH & Reverse,TGGTGAAGACGCCAGTGGA \\
\hline
\end{tabular}

NT-3, neurotrophin-3; BDNF, brain-derived neurotrophic factor; NSE, neuron-specific enolase.

Western blotting to detect BDNF, NT-3 and NSE protein expression. The transfected ADSCs were washed four times with PBS. Cells were lysed with $10 \mathrm{X}$ radioimmunoprecipitation assay buffer (Abcam) and protein concentration was determined using the Bicinchoninic Acid kit (Sigma-Aldrich). Protein samples $(50 \mu \mathrm{g})$ were separated by $10 \%$ SDS-PAGE (Sigma-Aldrich) and transferred to polyvinylidene fluoride membranes (Sigma-Aldrich). Non-specific reactivity was blocked using 5\% skim milk in tris-buffered saline containing Tween-20 (Sigma-Aldrich) for $60 \mathrm{~min}$ at room temperature. The membranes were then incubated with rabbit anti-rat BDNF (cat. no. ab6201), NT-3 (cat. no. ab65804) and NSE (cat. no. ab53025) antibodies (1:1,000 dilution; Abcam) overnight at $4{ }^{\circ} \mathrm{C}$, followed by incubation with goat anti-rabbit secondary antibodies (1:10,000 dilution; cat. no. ab6717; Abcam). $\beta$-actin antibodies (cat. no. ab129348; Abcam) served as an internal control. Image Lab 8.0 software (Bio-Rad Laboratories, Inc., Hercules, CA, USA) was used to measure the BDNF, NT-3, NSE and $\beta$-actin grey values, and the grey values of the proteins of interest were compared with the $\beta$-actin grey value, in order to determine the relative protein expression levels of BDNF, NT-3 and NSE. Western blotting was repeated five times.

Statistical analysis. Statistical analyses were conducted using SPSS 17.0 (SPSS Inc., Chicago, IL, USA). One-way analysis of variance was used for group comparisons. All values are presented as the mean \pm standard error and $\mathrm{P}<0.05$ was considered to indicate a statistically significant difference.

\section{Results}

ADSC morphology. Few cells had adhered to the bottom of the culture flask at $10 \mathrm{~h}$ following inoculation (Fig. 1A), however, at $72 \mathrm{~h}$ post-inoculation a large number of adherent cells were observed (Fig. 1B). At 8 days, the cells had reached 95\% confluence (Fig. 1C). There was no marked difference observed in the morphology of the third-generation cells, when compared with the primary cells (Fig. 1D).

ALP staining was conducted 2 weeks after osteogenic induction. In the experimental group, black particles were detected in the cytoplasm (Fig. 2A), whereas these particles 
A

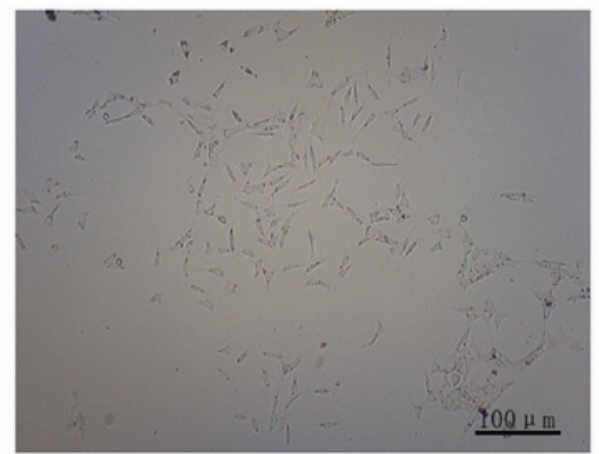

C

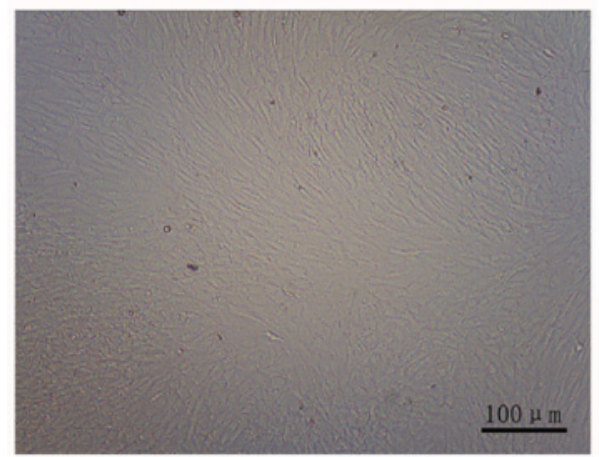

B

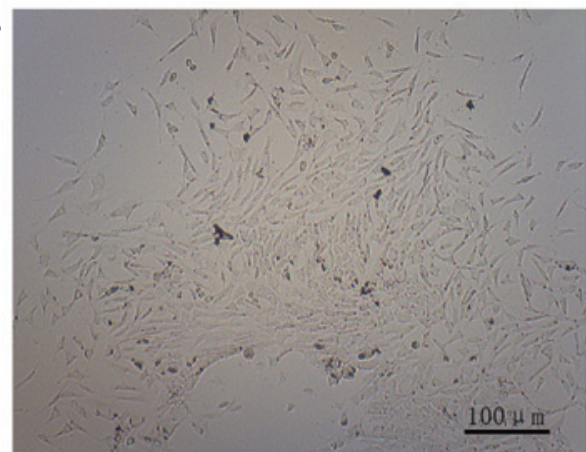

D

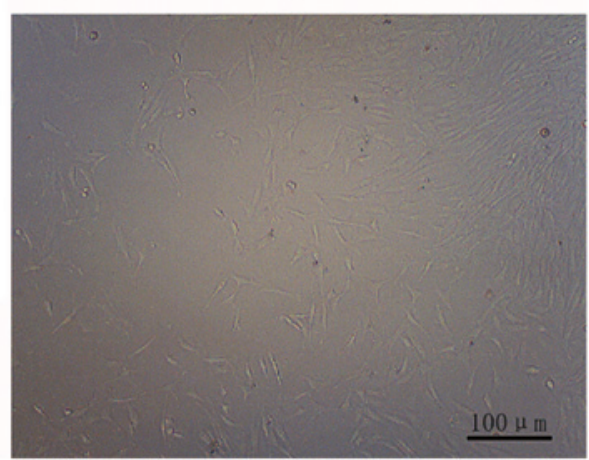

Figure 1. Observation of rat ADSC morphology (magnification, x100). (A) Rat ADSC morphology $10 \mathrm{~h}$ after inoculation. Primary culture after (B) $72 \mathrm{~h}$ and (C) 8 days. (D) Third-generation ADSC. ADSC, adipose-derived stem cell.

A

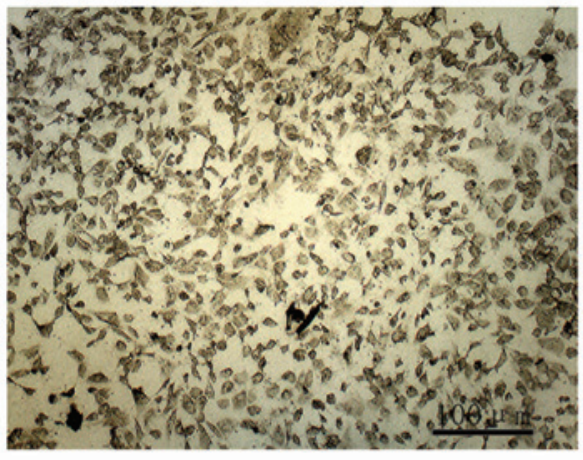

C

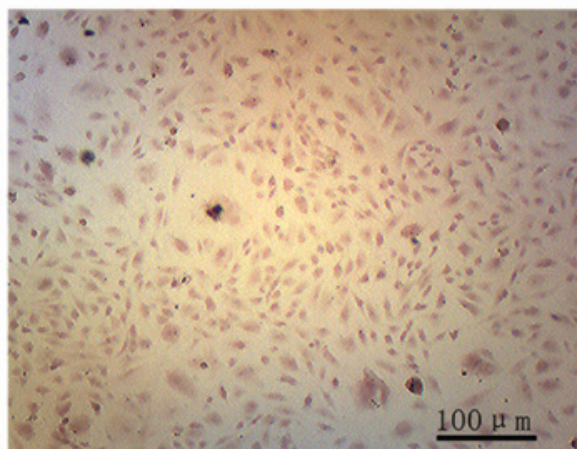

B

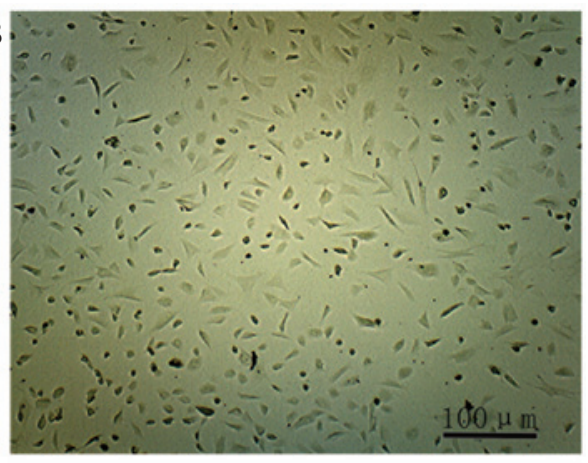

D

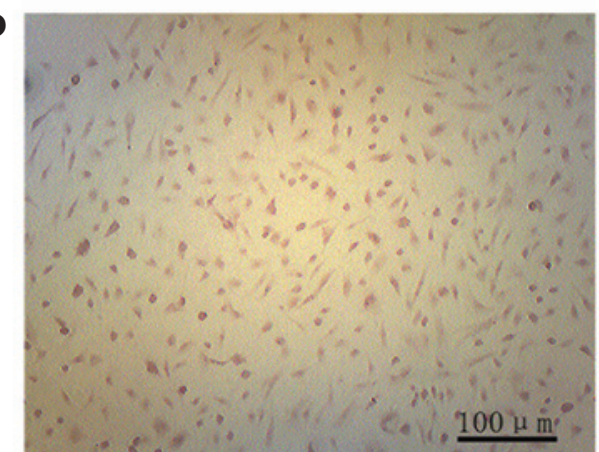

Figure 2. Staining of adipose-derived stem cells following osteogenic induction (magnification, x100). (A and B) Alkaline phosphatase staining 2 weeks after osteogenic induction in the (A) experimental and (B) control groups. (C and D) Alizarin red staining 4 weeks after osteogenic induction in the (C) experimental and (D) control groups.

were not observed in the control group (Fig. 2B). A total of 4 weeks after osteogenic induction alizarin red staining was conducted and calcium nodules were observed in the experimental group (Fig. 2C), whereas these nodules were not detected in the control group (Fig. 2D).
Selection of the optimum MOI value for ADSC transfection. ADSCs were transfected with lentiviruses at an MOI of 1,10 and 100 and the number of fluorescent cells gradually increased with the increasing MOI. When cells were transfected with a MOI of 100 , the fluorescent intensity was 
A

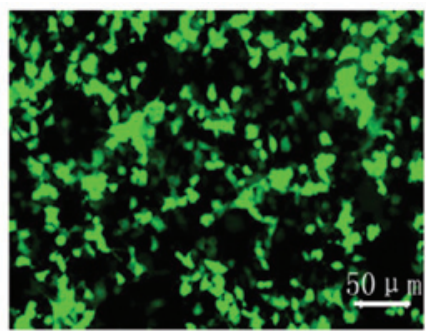

D

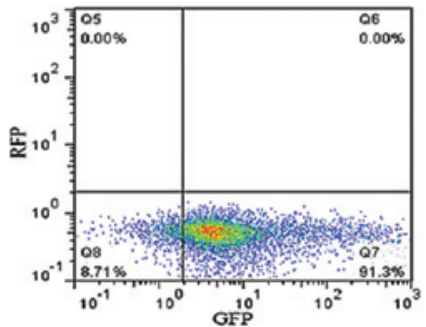

B

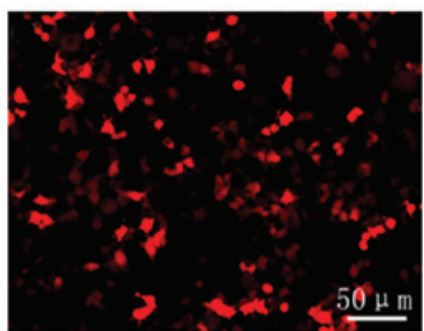

$\mathbf{E}$

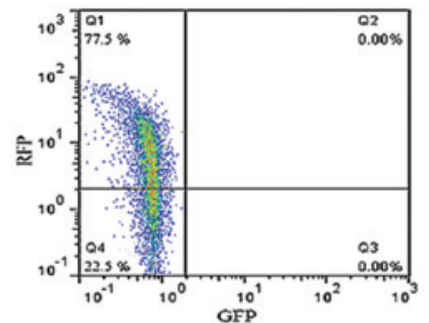

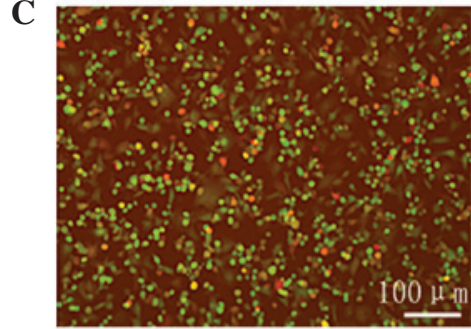

$\mathbf{F}$

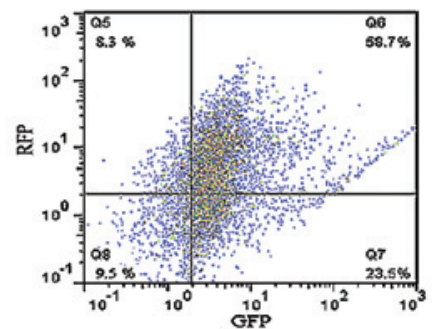

Figure 3. Determination of lentiviral transfection efficiency. (A-C) Fluorescence intensity of adipose-derived stem cells in the (A) Lenti-BDNF-GFP transfected group (magnification, x200), (B) Lenti-NT-3-RFP transfected group (magnification, x200) and the (C) Lenti-BDNF-GFP and Lenti-NT-3-RFP co-transfected group (magnification, x100). Number of (D) GFP-positive cells in the Lenti-BDNF-GFP transfected group, (E) RFP-positive cells in the Lenti-NT-3-RFP transfected group and (F) GFP- and RFP-positive cells in the Lenti-BDNF-GFP and Lenti-NT-3-RFP co-transfected group, as determined by flow cytometry. RFP, red fluorescent protein; GFP, green fluorescent protein; BDNF, brain-derived neurotophic factor; NT-3, neurotrophin-3.

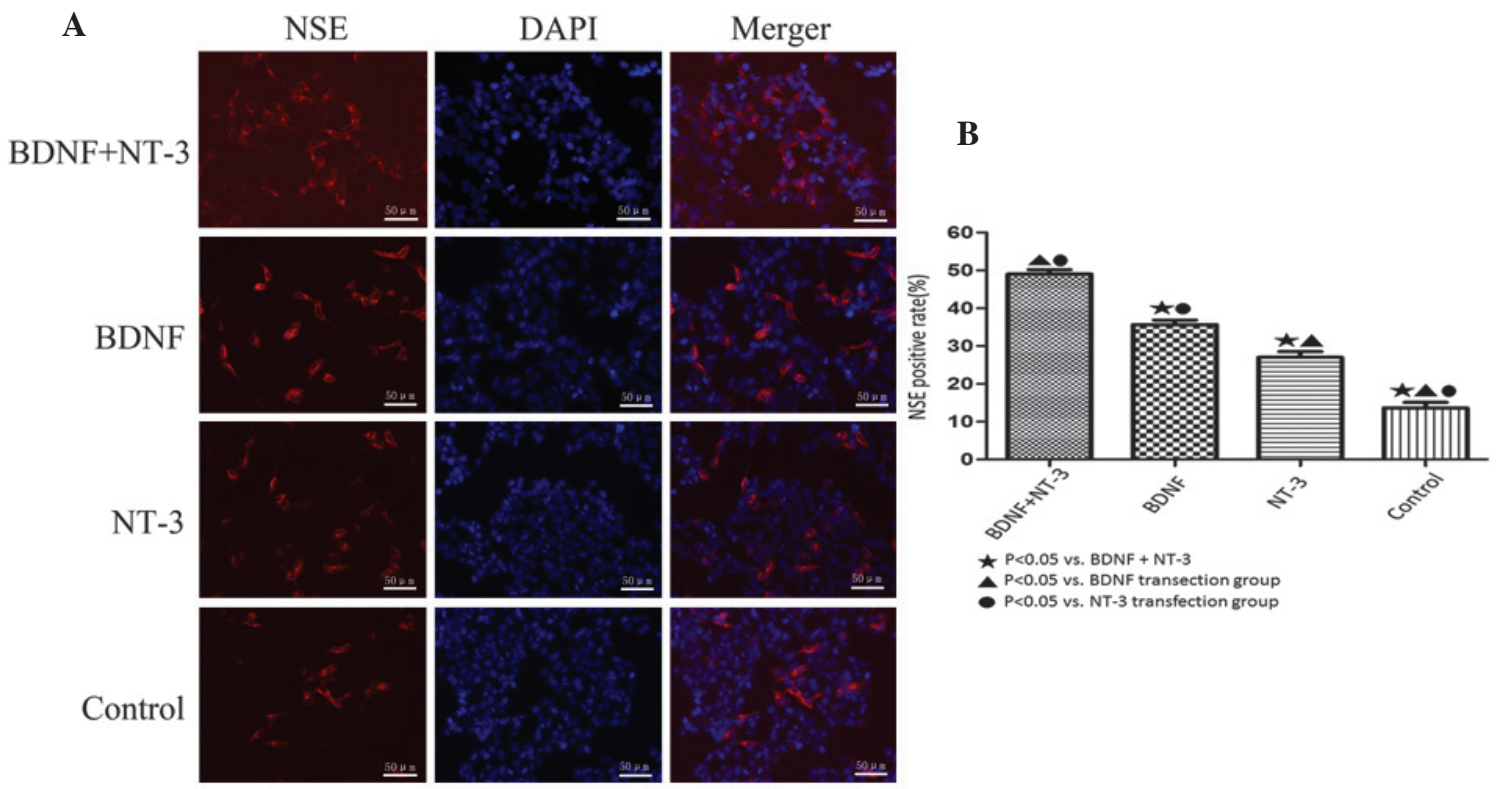

Figure 4. NSE immunofluorescent staining. (A) NSE-positive cells from each group (magnification, x200). (B) The percentage of NSE-positive cells in each group. Data are presented as the mean \pm standard error. NSE, neuron-specific enolase; BDNF, brain-derived neurotrophic factor; NT-3, neurotrophin-3.

significantly increased in the Lenti-BDNF-GFP transfected group (Fig. 3A), the Lenti-NT-3-RFP transfected group (Fig. 3B), and the Lenti-BDNF-GFP and Lenti-NT-3-GFP co-transfected group (Fig. 3C), concurrently a cell cytotoxic effect was detected. The optimum MOI was determined to be 100 , due to the higher transfection efficiency and slightly reduced cytopathogenicity. As determined by flow cytometry, the percentage of GFP-positive cells was $91.3 \%$ in the Lenti-BDNF-GFP transfected group (Fig. 3D), the percentage of RFP-positive cells was $77.5 \%$ in the Lenti-NT-3-RFP transfected group (Fig. 3E), and the percentage of GFP- and RFP-positive cells was 82.2 and $67.0 \%$, respectively in the
Lenti-BDNF-GFP and Lenti-NT-3-RFP co-transfected group (Fig. 3F).

Detection of NSE by immunofluorescent staining. ADSCs were induced to differentiate into neurons, and after 10 days NSE was most highly expressed in the BDNF and NT-3 co-transfected group (Fig. 4A), as compared with the other groups $(\mathrm{P}<0.05)$. The BDNF transfected group expressed the next highest levels of NSE, which were significantly lower than those in the co-transfected group $(\mathrm{P}<0.05)$, whereas the expression levels of NSE were lower in the NT-3 transfected group, as compared with the above groups; however, were 


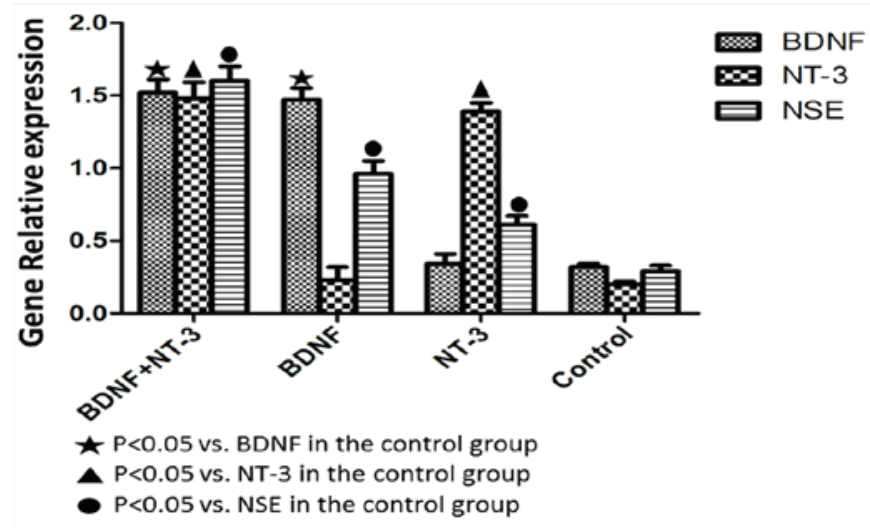

Figure 5. BDNF, NT-3 and NSE mRNA expression levels, as determined by reverse transcription-quantitative polymerase chain reaction 10 days after induction. Data are presented as the mean \pm standard error. BDNF, brain-derived neurotrophic factor; NT-3, neurotrophin-3; NSE, neuron-specific enolase.

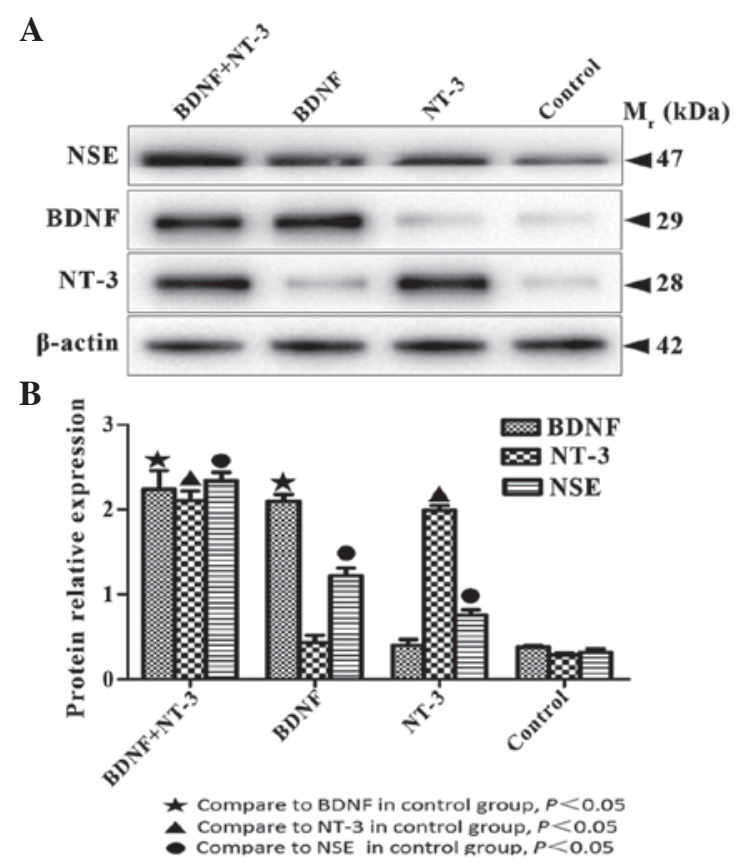

Figure 6. BDNF, NT-3 and NSE protein expression levels were examined by (A) western blotting 10 days after induction. (B) Quantification of western blotting. Data are presented as the mean \pm standard error. BDNF, brain-derived neurotrophic factor; NT-3, neurotrophin-3; NSE, neuron-specific enolase.

higher when compared with the control group $(\mathrm{P}<0.05$; Fig. 4B).

Detection of BDNF, NT-3 and NSE mRNA expression levels by $R T-q P C R$. BDNF, NT-3 and NSE mRNA expression levels were significantly higher in the experimental groups, as compared with the control group $(\mathrm{P}<0.05$; Fig. 5$)$. The mRNA expression levels of BDNF were significantly higher in the BDNF and NT-3 co-transfected group, as compared with the NT-3 transfected group $(\mathrm{P}<0.05)$. The mRNA expression levels of NT-3 were significantly higher in the BDNF and NT-3 co-transfected group, as compared with the BDNF transfected group $(\mathrm{P}<0.05)$. NSE mRNA expression levels were highest in the co-transfected group, followed by the BDNF transfected group, followed by the NT-3 transfected group $(\mathrm{P}<0.05)$.
Detection of BDNF, NT-3 and NSE protein expression levels by western blotting. BDNF, NT-3 and NSE protein expression levels were significantly higher in the experimental groups, as compared with those in the control group $(\mathrm{P}<0.05$; Fig. 6A). The protein expression levels of BDNF were significantly higher in the BDNF and NT-3 co-transfected group, as compared with the NT-3 transfected group $(\mathrm{P}<0.05)$. The protein expression levels of NT-3 protein were significantly higher in the BDNF and NT-3 co-transfected group, as compared with the BDNF transfect group $(\mathrm{P}<0.05)$. NSE protein expression levels were highest in the co-transfected group, followed by the BDNF transfected group, followed by the NT-3 transfected group $(\mathrm{P}<0.05$; Fig. 6B). The results of western blot analysis were consistent with the results of the immunofluorescent staining and RT-qPCR.

\section{Discussion}

SCI usually leads to permanent loss of function. Tissue engineering is a promising method that may be used to treat SCI, the underlying repair mechanisms of which are the secretion of neurotrophins from seed cells, and ultimately the differentiation of seed cells into neurons $(15,16)$. Therefore, the identification of a suitable seed cell that secretes neurotrophins and easily differentiates into neurons is important for the treatment of SCI.

ADSCs, which are adult SCs, have the advantage of convenience sampling, easy expansion, and possess the ability to differentiate into neurons; therefore, ADSCs are considered to be a promising seed cell in tissue engineering for the treatment of SCI (17). There are no specific surface antigens detected on ADSCs, therefore identification of ADSCs is usually based on organizational sources and their multiple differentiation potential (18). The present study obtained ADSCs by combining zymogen digestion with the differential velocity adherent method, and assumed that the separated cells were SCs, which therefore possess multilineage differentiation potential. The third-generation cells initially underwent osteogenic induction; after 2 weeks, calcium cobalt staining was positive and after 4 weeks, alizarin red staining was positive. These results indicated that the ADSCs had successfully differentiated into osteoblasts and possessed osteoblastic characteristics. In addition, the cells were isolated from adipose tissue, therefore it was reasonable to assume that the separated cells were ADSCs. Previous studies have demonstrated that ADSCs secrete neurotrophins, resulting in neuronal differentiation, however, this ability is limited. BDNF and NT-3 are important neurotrophins, which possess superior properties, when compared with other types of neurotrophic factors, in the maintenance of neuronal survival, inhibition of cell apoptosis, and promotion of SC differentiation into neurons $(19,20)$. In order to improve the neurotrophin secretory ability of ADSCs, two types of lentivirus were constructed in the present study: Lenti-BDNF-GFP and Lenti-NT-3-RFP. The lentiviruses were transfected into third-generation ADSCs at various MOIs. Transfection with an MOI of 100 for $72 \mathrm{~h}$ was associated with improved transfection efficiency and reduced cytopathogenicity. Furthermore, flow cytometry was used to determine transfection efficiency; the percentage of 
GFP-positive cells was $91.3 \%$ in the Lenti-BDNF-GFP transfected group, the percentage of RFP-positive cells was $77.5 \%$ in the Lenti-NT-3-RFP transfected group, and the percentage of GFP- and RFP-positive cells was 82.2 and $67.0 \%$, respectively, in the Lenti-BDNF-GFP and Lenti-NT-3-RFP co-transfected group. The transfected cells were subsequently induced to differentiate into neurons, and after 10 days the expression levels of BDNF and NT-3 were detected. The BDNF and NT-3 co-transfected group expressed BDNF and NT-3 mRNA and protein at high levels. The BDNF transfected group expressed high levels of BDNF and low levels of NT-3, the NT-3 transfected group expressed high levels of NT-3 and low levels of BNDF, and the control group expressed BDNF and NT-3, however at significantly lower levels than the transfected groups. Furthermore, NSE expression levels were determined using immunofluorescent staining, RT-qPCR and western blotting, the results of which demonstrated that NSE was most highly expressed in the BDNF and NT-3 co-transfected group, followed by the BDNF transfected group, followed by the NT-3 transfected group.

A previous study demonstrated that BDNF and NT-3 belong to a group of neurotrophins that are targeted by the Trk receptor, which is divided into three types: TrkA, TrkB and TrkC (21). BDNF is targeted by TrkB, and NT-3 is targeted by TrkC, however NT-3 may occasionally bind to TrkA and TrkB $(22,23)$. Based on these previous findings and the results of the present study, it may be hypothesized that BDNF and NT-3 co-transfected cells easily differentiate into neurons due to the increased secretion of BDNF and NT-3, which bind TrkB and TrkC resulting in the formation of an active dimer. Via the process of Trk receptor phosphorylation cell signals may be transmitted that promote the differentiation of ADSCs into neurons. Another hypothesis reported by Hapner et al (24) is that BDNF and NT-3 may prompt the expression of the Trk receptor, which may promote SC neuronal differentiation. An additional hypothesis is that BDNF and NT-3 exert a synergistic effect that may promote the differentiation of ADSCs into neurons. In the present study, the BDNF and NT-3 transfected groups were able to secrete BDNF and NT-3, and the number of NSE-positive cells in these groups was higher, as compared with the control group, thus suggesting that ADSCs modified by BDNF and NT-3 may easily differentiate into neurons. In addition, when compared with the NT-3 transfected group, ADSCs modified by BDNF more easily differentiated into neurons; however, the mechanism behind this finding is unclear. The present study hypothesized that BDNF and NT-3 may affect the differentiation of ADSCs into neurons, with BDNF exerting a key role and NT-3 exerting a secondary role, which may be important in axon growth, as demonstrated by Francis et al (25). The results of the present study demonstrate that ADSCs modified by BDNF and NT-3 differentiate into neurons more easily.

In conclusion, the present study examined the effects of BDNF and NT-3 overexpression on neuronal differentiation of rat ADSCs. The results indicate that BDNF and NT-3 exert a synergistic regulatory effect on ADSC neuronal differentiation, and that ADSCs may easily differentiate into neurons after being modified by BDNF and NT-3. ADSCs may therefore be suitable seed cells for use in tissue engineering for the treatment of SCI. Our future research will be focused on developing an in-depth understanding regarding cell signaling following binding of BDNF and NT-3 to the Trk receptor. Thus, the present study provides insight into the use of tissue engineering technology for future treatment of SCI.

\section{Acknowledgements}

The authors of the present study would like to thank Professor Ying Mao (Xi'an Jiaotong University) who provided guidance during the experiment.

\section{References}

1. Hayashi T, Kawano O, Sakai H, Ideta R, Ueta T, Maeda T, Mori E, Yugue I, Takao T, Masuda M, et al: The potential for functional recovery of upper extremity function following cervical spinal cord injury without major bone injury. Spinal Cord 51: 819-822, 2013

2. Cadotte DW and Fehlings MG: Spinal cord injury: A systematic review of current treatment options. Clin Orthop Relat Res 469: 732-741, 2011.

3. Mortazavi MM, Harmon OA, Adeeb N, Deep A and Tubbs RS: Treatment of spinal cord injury: A review of engineering using neural and mesenchymal stem cells. Clin Anat 28: 37-44, 2015.

4. Ribeiro-Samy S, Silva NA, Correlo VM, et al: Development and characterization of a PHB-HV-based 3D scaffold for a tissue engineering and cell-therapy combinatorial approach for spinal cord injury regeneration. Macromol Biosci 13: 1576-1592, 2013.

5. Kanno H, Pressman Y, Moody A, Berg R, Muir EM, Rogers JH, Ozawa H, Itoi E, Pearse DD and Bunge MB: Combination of engineered Schwann cell grafts to secrete neurotrophin and chondroitinase promotes axonal regeneration and locomotion after spinal cord injury. J Neurosci 34: 1838-1855, 2014.

6. Abbasi M, Salehi M, Pasbakhsh P and Sobhani A: Repair of spinal cord injury by co-transplantation of embryonic stem cell-derived motor neuron and olfactory ensheathing cell. J Stem Cells Regen Med 6: 81, 2010.

7. Ji W, Hu S, Zhou J, Wang G, Wang K and Zhang Y: Tissue engineering is a promising method for the repair of spinal cord injuries (Review). Exp Ther Med 7: 523-528, 2014.

8. Kapur SK and Katz AJ: Review of the adipose derived stem cell secretome. Biochimie 95: 2222-2228, 2013.

9. Liqing Y, Jia G, Jiqing C, Ran G, Fei C, Jie K, Yanyun W and Cheng Z: Directed differentiation of motor neuron cell-like cells from human adipose-derived stem cells in vitro. Neuroreport 22: 370-373, 2011.

10. Liu H, Chu Y and Lou G: Fiber-modified adenovirus can mediate human adipose tissue-derived mesenchymal stem cell-based anti-angiogenic gene therapy. Biotechnol Lett 32: 1181-1188, 2010.

11. Liu W, Lu G, Wang B, Ma Z and Li Y: Transfection of BDNF gene promotes bone mesenchymal stem cells to differentiate into neuron-like cells. Zhong Nan Da Xue Xue Bao Yi Xue Ban 37: 441-446, 2012 (In Chinese).

12. Gao H, Wei M, Wang Y, Wu X and Zhu T: Differentiation of GDNF and NT-3 dual gene-modified rat bone marrow mesenchymal stem cells into enteric neuron-like cells. J Huazhong Univ Sci Technolog Med Sci 32: 87-91, 2012.

13. Ji W, Zhang Y and Hu S: Biocompatibility study of a silk fibroin-chitosan scaffold with adipose tissue-derived stem cells in vitro. Exp Ther Med 6: 513-518, 2013.

14. Livak KJ and Schmittgen KD: Analysis of relative gene expression data using real-time quantiative PCR and the 2(-Delta Delta C(T)) Method. Methods 25: 402-408, 2001.

15. Madigan NN, McMahon S, O'Brien T, Yaszemski MJ and Windebank AJ: Current tissue engineering and novel therapeutic approaches to axonal regeneration following spinal cord injury using polymer scaffolds. Respir Physiol Neurobiol 169: 183-199, 2009.

16. Silva NA, Salgado AJ, Sousa RA, Oliveira JT, Pedro AJ, Leite-Almeida H, Cerqueira R, Almeida A, Mastronardi F, Mano JF, et al: Development and characterization of a novel hybrid tissue engineering-based scaffold for spinal cord injury repair. Tissue Eng Part A 16: 45-54, 2010. 
17. Zhou J, Lu P, Ren H, Zheng Z, Ji J, Liu H, Jiang F, Ling S, Heng BC, $\mathrm{Hu} \mathrm{X}$ and Ouyang H: $17 \beta$-estradiol protects human eyelid-derived adipose stem cells against cytotoxicity and increases transplanted cell survival in spinal cord injury. J Cell Mol Med 18: 326-343, 2014.

18. Ren Y, Wu H, Ma Y, Cang M, Wang R and Liu D: Isolation, cultivation and identification of adipose-derived stem cell in bovines. Sheng Wu Gong Cheng Xue Bao 26: 1645-1651,2010 (In Chinese).

19. Gibbons A, Wreford N, Pankhurst J and Bailey K: Continuous supply of the neurotrophins BDNF and NT-3 improve chick motor neuron survival in vivo. Int J Dev Neurosci 23: 389-396, 2005.

20. Wang Y, Gu J, Wang J, Feng X, Tao Y, Jiang B, He J, Wang Q, Yang J, Zhang S, et al: BDNF and NT-3 expression by using glucocorticoid-induced bicistronic expression vector pGC-BDNF-IRES-NT3 protects apoptotic cells in a cellular injury model. Brain Res 1448: 137-143, 2012.

21. Sobue G, Yamamoto M, Doyu M, Li M, Yasuda T and Mitsuma T: Expression of mRNAs for neurotrophins (NGF, BDNF, and NT-3) and their receptors (p75NGFR, trk, trkB, and trkC) in human peripheral neuropathies. Neurochem Res 23: 821-829, 1998.
22. Islam O, Loo TX and Heese $\mathrm{K}$ : Brain-derived neurotrophic factor (BDNF) has proliferative effects on neural stem cells through the truncated TRK-B receptor, MAP kinase, AKT and STAT-3 signaling pathways. Curr Neurovasc Res 6: 42-53, 2009.

23. Zhang YQ, Zeng X, He LM, Ding Y, Li Y and Zeng YS: NT-3 gene modified Schwann cells promote TrkC gene modified mesenchymal stem cells to differentiate into neuron-like cells in vitro. Anat Sci Int 85: 61-67, 2010.

24. Hapner SJ, Nielsen KM, Chaverra M, Esper RM, Loeb JA and Lefcort F: NT-3 and CNTF exert dose-dependent, pleiotropic effects on cells in the immature dorsal root ganglion: Neuregulin-mediated proliferation of progenitor cells and neuronal differentiation. Dev Biol 297: 182-197, 2006.

25. Francis N, Farinas I, Brennan C, Rivas-Plata K, Backus C, Reichardt L and Landis S: NT-3, like NGF, is required for survival of sympathetic neurons, but not their precursors. Dev Biol 210: 411-427, 1999. 